\title{
MRI Findings at Term-Corrected Age and Neurodevelopmental Outcomes in a Large Cohort of Very Preterm Infants
}

\author{
(D) S. Arulkumaran, (DN. Tusor, (D) A. Chew, (D). Falconer, (D) N. Kennea, (D). Nongena, (D).V. Hajnal, (D) S.J. Counsell,
}

(D) M.A. Rutherford, and (D) A.D. Edwards

\begin{abstract}
BACKGROUND AND PURPOSE: Brain MR imaging at term-equivalent age is a useful tool to define brain injury in preterm infants. We report pragmatic clinical radiological assessment of images from a large unselected cohort of preterm infants imaged at term and document the spectrum and frequency of acquired brain lesions and their relation to outcomes at 20 months.
\end{abstract}

MATERIALS AND METHODS: Infants born at <33 weeks' gestation were recruited from South and North West London neonatal units and imaged in a single center at 3T at term-equivalent age. At 20 months' corrected age, they were invited for neurodevelopmental assessment. The frequency of acquired brain lesions and the sensitivity, specificity, and negative and positive predictive values for motor, cognitive, and language outcomes were calculated, and corpus callosal thinning and ventricular dilation were qualitatively assessed.

RESULTS: Five hundred four infants underwent 3T MR imaging at term-equivalent age; 477 attended for assessment. Seventy-six percent of infants had acquired lesions, which included periventricular leukomalacia, hemorrhagic parenchymal infarction, germinal matrix-intraventricular hemorrhage, punctate white matter lesions, cerebellar hemorrhage, and subependymal cysts. All infants with periventricular leukomalacia, and $60 \%$ of those with hemorrhagic parenchymal infarction had abnormal motor outcomes. Routine 3T MR imaging of the brain at term-equivalent age in an unselected preterm population that demonstrates no focal lesion is $45 \%$ sensitive and $61 \%$ specific for normal neurodevelopment at 20 months and $17 \%$ sensitive and $94 \%$ specific for a normal motor outcome.

CONCLUSIONS: Acquired brain lesions are common in preterm infants routinely imaged at term-equivalent age, but not all predict an adverse neurodevelopmental outcome.

ABBREVIATIONS: BSID-III = Bayley Scales of Infant Development, Version III; GMFCS = Gross Motor Function Classification System; GMH-IVH = germinal matrix hemorrhage-intraventricular hemorrhage; HPI = hemorrhagic parenchymal infarction; IMD $=$ index of multiple deprivation; $\mathrm{M}$ - $\mathrm{CHAT}=\mathrm{Modified}$ Checklist for Autism in Toddlers; PLIC = posterior limb of the internal capsule; PMA = postmenstrual age; PVL = periventricular leukomalacia; PWML = punctate white matter lesions; TEA = term-equivalent age

$\mathbf{P}$ reterm birth, defined as $<37$ completed weeks' gestation, accounts for $9.6 \%$ of live births globally ${ }^{1}$ and represents the largest single cause of neonatal morbidity and mortality worldwide. Advances in obstetric and neonatal intensive care have resulted in a decrease in neonatal mortality; however, the

\section{Received February 4, 2020; accepted after revision May 19}

From the Centre for the Developing Brain (S.A., N.T., A.C., S.F., J.V.H., S.J.C., M.A.R., A.D.E.), School of Biomedical engineering and Imaging Sciences, King's College London and Evelina London Children's Hospital, London, UK; Neonatal Unit (N.K.), St. George's Hospital, London, UK; and Division of Clinical Sciences (P.N.), Imperial College London, London, UK.

Mary A. Rutherford and A. David Edwards are joint final authors.

The views and opinions expressed by authors in this publication are those of the authors and do not necessarily reflect those of the National Health Service, the National Institute for Health Research, the Medical Research Council, Central Commissioning Facility, National Institute for Health Research Evaluation, Trials and Studies Coordinating Center, the Program Grants for Applied Research Program, or the Department of Health.
This work presents independent research commissioned by the National Institute for Health Research. The project was part of a program of research funded by the National Institute for Health Research Program Grants for Applied Research Program (RP-PG-0707-10154). The work was also supported by the Medical Research Council Center for Neurodevelopmental Disorders, which provided a PhD studentship for S.A. (MR/K006355/1), as well as the Wellcome Trust, Engineering and Physical Sciences Research Council Medical Engineering Center, the National Institute for Health Research Biomedical Research Centers at Guy's and St Thomas' National Health Service Foundation Trust (STR1110011), and King's College London and Imperial College Healthcare Trust.

Paper previously presented as a poster at: Academy of Medical Sciences Scientific Meeting: The Developing Brain in Health and Disease, April 25, 2019; Oxford, UK.

Please address correspondence to Sophie Arulkumaran, FRCR, Centre for the Developing Brain, School of Biomedical engineering and Imaging Sciences, King's College London, and Evelina Children's Hospital, London SEI 7EH, UK; e-mail: sg-baker@doctors.org.uk

\footnotetext{
- Indicates open access to non-subscribers at www.ajnr.org

$\equiv$ Indicates article with supplemental on-line tables.

Indicates article with supplemental on-line photos.

http://dx.doi.org/10.3174/ajnr.A6666
}

AJNR Am J Neuroradiol 41:1509-16 Aug 2020 www.ajnr.org 
incidence of major morbidity has remained constant. ${ }^{2}$ The most immature infants are at greatest risk of complications, with neurodevelopmental impairment in up to $50 \%$ of infants born at $<26$ weeks' gestation. ${ }^{3}$

MR imaging is increasingly used in the preterm population to complement cranial sonography to improve prognostic information and inform current clinical and future supportive care. In addition, the information received from neuroimaging, in particular MR imaging, can reduce maternal anxiety surrounding their preterm infant. ${ }^{4}$

MR imaging provides a safe technique to assess preterm brain development, with numerous studies describing typical acquired injuries and advanced MR imaging techniques demonstrating widespread alterations in development associated with premature exposure to the extrauterine environment. ${ }^{5} \mathrm{MR}$ imaging scoring systems have been developed for both specific lesions and to characterize the more global injury following preterm birth: Edwards et $\mathrm{al}^{4}$ published data using a scoring system to demonstrate an association between cranial sonographic findings, moderate-to-severe brain injury on term-equivalent age (TEA) MR imaging, and poor motor outcomes at 2 years. Tusor et $\mathrm{al}^{6}$ combined structural imaging with tractography to demonstrate a relationship between the number and site of punctate white matter lesions (PWML) and adverse motor development; however, the prognostic advantage of MR imaging in these contexts was modest. ${ }^{4,6}$

While MR imaging has advanced our understanding of preterm brain injury, the ability to predict neurodevelopmental outcome in an individual remains elusive, with previous studies reporting individual findings in relation to neurodevelopmental outcomes in small cohorts or group differences in larger cohorts. $^{7-9}$ In addition, discrepancies occur due to variable classifications of lesions, and in many, MR imaging will have been performed in response to abnormalities seen on sonography. In this study, we depart from scoring systems and document the frequency and spectrum of lesions encountered in a large population-based cohort of preterm infants routinely imaged at TEA and relate these to neurodevelopmental outcomes, aiming to aid the reporting of these complex cases without scoring systems, which often prove impractical for everyday radiological practice.

This study aimed to document the spectrum and frequency of acquired brain lesions in a large cohort of infants born very preterm (<33 weeks' completed gestation) and imaged at TEA (3844 weeks' post menstrual age [PMA]) as routine, in a single centre using $3 \mathrm{~T}$ MR imaging and to compare image findings with standardized neurodevelopmental outcomes at 20 months' corrected age.

\section{MATERIALS AND METHODS}

Ethics approval was granted by the Hammersmith Research Ethics Committee (09/H0707/98) and parental consent was given for all examinations.

\section{Study Design}

The data were obtained from the Evaluation of MR Imaging to Predict Neurodevelopmental Impairment in Preterm Infants
(ePrime) study, which was a randomized, controlled trial comparing MR imaging and cranial sonography in the care of preterm infants. ${ }^{4}$ Infants born at $<33$ weeks' completed gestation were prospectively recruited from the South and North West London perinatal networks between 2009 and 2013. Informed written consent was obtained for $3 \mathrm{~T}$ brain MR imaging at termequivalent age, all performed at a single institution.

Patient and clinical characteristics were obtained from the SEND database (a UK national standardized neonatal database) and discussion with parents. Gestational age was based on the earliest antenatal sonogram, and PMA at imaging was derived from this. Socioeconomic status was measured using the index of multiple deprivation (IMD), a score based on the mother's postal address: higher scores denote more deprived areas.

\section{MR Imaging Protocol}

A neonatal specific MR metal check was performed, and the infant was then either fed and swaddled or sedated with oral chloral hydrate. Earplugs and neonatal ear muffs were used. Continuous pulse oximetry, electrocardiography, and temperature monitoring remained in situ throughout the study. All imaging was performed on a 3T MR scanner (Philips Medical Systems, Best, The Netherlands) using an 8-channel head coil. A 3D T1 (MPRAGE) sequence was acquired in the sagittal plane and reconstructed into orthogonal planes $(\mathrm{TR}=17 \mathrm{~ms}$, $\mathrm{TE}=$ $4.6 \mathrm{~ms}$, resolution $=0.8 \times 0.8 \times 0.8 \mathrm{~mm})$, and an axial dualecho $\mathrm{T} 2$ scan with sensitivity encoding $(\mathrm{TR}=15,000 \mathrm{~ms}$, $\mathrm{TE}=$ $160 \mathrm{~ms}$, resolution $=0.86 \times 0.86 \times 2 \mathrm{~mm}$ ) was performed. Where problematic motion existed, a single-shot $\mathrm{T} 2$ sequence with overlapping slices was acquired $(\mathrm{TR}=$ shortest, $\mathrm{TE}=160$ $\mathrm{ms}$, resolution $=0.86 \times 0.86 \times 2 \mathrm{~mm})$.

\section{MR Imaging Analysis}

All images were analyzed by readers with specific preterm neonatal brain MR imaging experience (S.A. 1 year; N.T. 5 years; M.A.R. $>20$ years), blinded to outcomes and clinical details except gestational age and PMA.

\section{Imaging Classifications}

Typical Preterm Brain Phenotype. Development of the brain is known to be altered by preterm delivery. Studies have previously documented reduced cortical folding, diffuse excessive high T2 signal intensity in the white matter, and a scaphocephalic head shape (Fig 1). ${ }^{10}$ We graded corpus callosal thickness as either normal, focal, or global thinning (On-line Fig 1). Ventricular dilation was graded as absent, moderate, or global enlargement of a moderate-to-severe nature (On-line Fig 2).

Myelination. Myelin within the posterior limb of the internal capsule (PLIC) is readily identifiable in the neonatal brain; absence or marked asymmetry was considered pathological (Figs 1-3).

\section{Classification of Lesions}

Germinal Matrix Hemorrhage-Intraventricular Hemorrhage. The germinal matrix, which is seen normally, involutes with age; only remnants are seen in the caudothalamic notch and roof of the temporal horns after 32 weeks. An irregular contour to a 


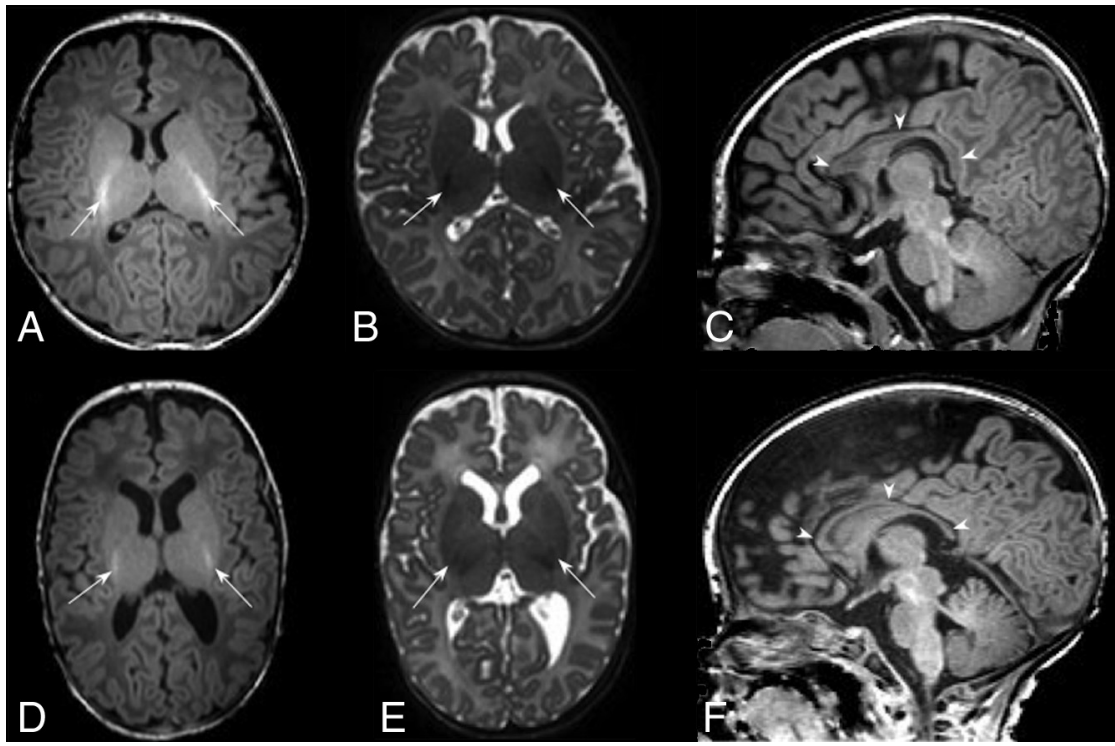

FIG 1. Typical MR imaging brain appearances of term-born and preterm infants, at TEA. Axial TT$(A)$ and T2- $(B)$ and sagittal T1 (C)-weighted images of a term-born infant (40 weeks' gestational age) imaged at 44 weeks PMA. Note the high-T1/low-T2 signal within the PLIC from the myelin (arrows, $A$ and $B$ ); the complex cortical folding; small, symmetric ventricles; and the typical appearance of a normal corpus callosum (arrowheads, $C$ ). By comparison, $D-F$ are comparable images obtained from an infant born at $25+2$ weeks' gestational age and imaged at $42+4$ weeks' PMA. This infant had no acquired focal lesions however, note the scaphocephaly, reduced cortical folding, globally thinned corpus callosum (arrowheads, F), mildly dilated ventricles, and diffuse high-T2/low-T1 signal in the white matter compared with the term infant. There is grossly normal myelination in the PLIC (arrows, $D$ and $E$ ).

subependymal area of low T2 signal or accompanying intraventricular hemorrhage was classified as germinal matrix hemorrhage-intraventricular hemorrhage (GMH-IVH), knowing that hemorrhage may be inconspicuous at TEA (Fig 4).

Hemorrhagic Parenchymal Infarction. Once fully evolved, hemorrhagic parenchymal infarction (HPI) appears as a focal outpouching of the ventricular contour (usually unilateral), often with a low T2 signal element, consistent with previous hemorrhage (Fig 2).

Periventricular Leukomalacia. The "classic" appearance of periventricular leukomalacia (PVL) is of multiple bilateral periventricular cysts in a symmetric distribution, initially distinct from the ventricle (solitary or unilateral cysts are more likely to be venous infarcts or connatal cysts). By TEA, the cysts may no longer be apparent; therefore, we used the following criteria: residual bilateral periventricular cysts and dilated/angulated posterior aspects of the lateral ventricles with associated white matter volume loss, often accompanying thalamic atrophy and abnormal myelination (Fig 3). ${ }^{11}$

Punctate White Matter Lesions. PWML are small areas of white matter injury with no reliable sonographic correlate. ${ }^{6}$ PWML were defined as small foci of high T1 signal in the white matter, less often visualized on T2 sequences (On-line Fig 3). Conspicuity decreases with time; therefore, interpretation was based on the understanding that the original lesion load was likely higher. PWML in this cohort have been comprehensively reported by Tusor et al. ${ }^{6}$

Subependymal Cysts. Subependymal cysts are thin-walled, typically occurring at the caudothalamic notch, often but not always the sequelae of germinal matrix hemorrhage (On-line Fig 4). Additional findings, such as temporal pole cysts, may suggest cytomegalovirus infection.

Cerebellar Hemorrhage. We defined cerebellar hemorrhage as small $(<5$ $\mathrm{mm})$ or large $(>5 \mathrm{~mm})$. By TEA, there may be atrophy with minimal hemorrhage. The germinal matrix is sited on the outer surface of the cerebellum; therefore, hemorrhages may involve the cerebellar cortex (On-line Fig 5).

Unclassified Lesions. Unclassified lesions encompassed findings that did not conform to the above lesion definitions. These were considered major if associated with lobar or multiple areas of tissue loss.

Pseudocysts are specifically discussed because they require distinction from subependymal cysts and periventricular leukomalacia (PVL) (On-line Fig 6). They are located within the caudate head and inferior to the external angle of the ventricle (PVL is superior) and anterior to the foramen of Monro (subependymal cysts are posterior). ${ }^{12}$ Pseudocysts decrease in size with time.

\section{Neurodevelopmental Follow-Up}

All participants were invited for neurodevelopmental assessment at 20 months' corrected age, which comprised a Bayley Scales of Infant Development, Version III (BSID-III), ${ }^{13}$ a Gross Motor Function Classification System (GMFCS) score, and parentreported Modified Checklist for Autism in Toddlers (MCHAT) ${ }^{14}$ The BSID-III and GMFCS were performed by a clinician blinded to the clinical history and imaging results. Abnormal development was defined as a BSID-III score of $<85$ in any domain ${ }^{15}$ and/or a GMFCS score of $\geq 2$ (indicative of moderate-to-severe gross motor impairment). An M-CHAT score of $\geq 3$ for any item (or $\geq 2$ for critical items) was considered a positive screen for autism/pervasive developmental disorder. ${ }^{14}$

\section{Statistical Analysis}

Statistical analyses were performed using SPSS Version 25 (IBM). BSID-III outcomes were dichotomized into abnormal/normal; and specificity, sensitivity, and negative and positive predictive values were calculated. Continuous variables were analyzed using either an independent $t$ test or a Mann-Whitney $U$ test, depending on the distribution of the data and adjusted for multiple comparisons using the Bonferroni method. For statistical analysis, 
PVL, HPI, >10 PWML, and any substantial "unclassified” lesion were classified as major. Analysis of the patient characteristics by lesion type was considered a secondary outcome and, therefore, not adjusted for multiple comparisons.

\section{RESULTS}

Of the 512 participants, 1 child was excluded because the imaging findings were typical of a genetic diagnosis (pontocerebellar hypoplasia). MR imaging was not possible in 6 (1\%), 27 (5\%) did not attend for neurodevelopmental assessment, and 1 child died of sudden infant death syndrome at 2 months (with a few PWML on MR imaging). This left 477 (93\%) infants with MR imaging and neurodevelopmental follow-up. There was no difference in the presence of lesions on MR imaging for those who did/did not attend for
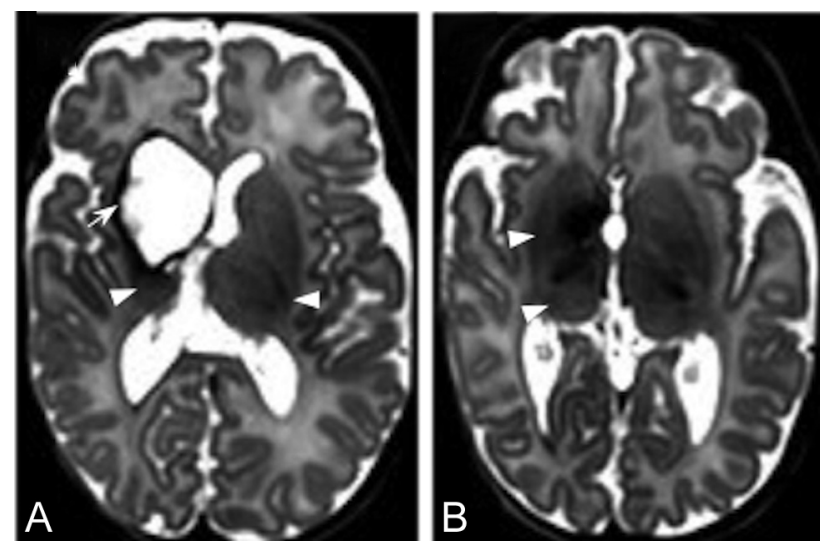

FIG 2. HPI. T2-weighted MR images through the mid $(A)$ and low $(B)$ basal ganglia level in an infant born at 29 weeks and imaged at $44+6$ weeks. There is right-sided HPI with formation of a porencephalic cyst lined with low signal intensity consistent with previous hemorrhage (arrow, A), a paucity of low T2 signal myelin in the right PLIC compared with the left (arrowheads, A), and ipsilateral basal ganglia and thalamic atrophy (arrowheads, B). This infant had a motor impairment at 20 months. neurodevelopmental follow-up $(P=.10)$. Fifty-six percent $(n$ $=272$ ) of infants were developing normally at 20 months.

\section{Patient Characteristics}

The patient characteristics are summarized in On-line Table 1. The median gestational age was 30 weeks, and PMA was 42.7 weeks. One hundred infants were imaged beyond 44 weeks, and one, at 37 weeks. Thirty-two percent $(n=162)$ of infants were born from a multiple pregnancy ( $n=24$ triplets, $n=3$ quadruplets, $n=135$ twins).

\section{MR Imaging Lesion Analysis}

Of the 504 participants who had MR imaging, 45 (9\%) had motion artifacts and $11(2 \%)$ had an incomplete set of sequences. Seventy-nine percent $(n=403)$ of participants had sedation, of which $8 \%$ had motion artifacts, compared with $16 \%$ of those who did not receive sedation $(P=.07)$.

Of the 504 infants imaged, $76 \%(n=383)$ had lesions and $41 \%(n=208)$ had lesions in $>1$ category. Thirteen percent $(n=68)$ of infants had major lesions. Infants born of a multiple pregnancy were not more likely than singletons to have an abnormal BSID-III score or lesions on their MR imaging.

A summary of the rate of abnormal outcomes, sensitivity, specificity, and positive and negative predictive values for each lesion type is tabulated in the Table, On-line Table 2, and On-line Figs 7 and 8 .

\section{Outcome and MR Imaging: Brains with No Focal Lesions}

Twenty-four percent $(n=120)$ of infants had no focal lesion; $58 \%$ of this group achieved normal outcomes. Thus, $14 \%$ of the entire cohort had no focal brain lesions on MR imaging and normal neurodevelopment at 20 months' corrected age. These infants were born at a later gestation (30.1 versus 29.6 weeks, $P=.02$ ), ventilated for shorter periods (1.5 versus 3.2 days, $P<.001)$, and were less likely to have evidence of infection ( $8 \%$ versus $16 \% P=.03$ ) compared to infants with

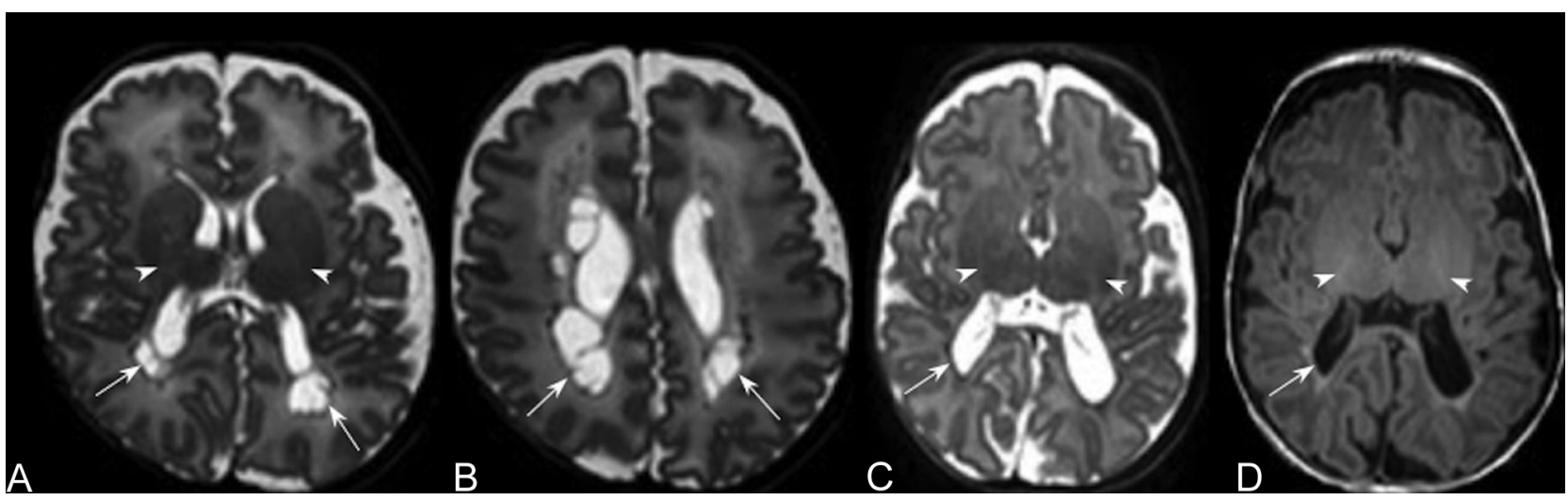

FIG 3. PVL. T2-weighted images in the axial plane at the level of the basal ganglia $(A)$ and the corona radiata $(B)$ of an infant born at $29+5$ weeks' and imaged at $39+1$ weeks' PMA. There are cysts in the periventricular white matter bilaterally (arrows, $A$ and $B$ ) accompanied by an absence of high-T1/low-T2 signal in the PLIC, denoting an absence of myelin (arrowheads, A). More commonly, cysts are not seen at term age. T2- (C) and T1-weighted $(D)$ images in the axial plane at the level of the basal ganglia and PLIC in an infant born at $29+3$ weeks' and imaged at $40+2$ weeks' PMA show mild angulation and dilation of the posterior horns of the lateral ventricles and high-T1/low-T2 signal in the periventricular white matter (arrows, $C$ and $D$ ), secondary to white matter volume loss (note that the sulci approximate the ventricular surface), similarly accompanied by a lack of myelin in the PLIC (arrowheads, C and D), features that are typical of noncystic PVL. 
focal lesions. The IMD score was significantly higher in infants with no focal lesion and an abnormal outcome compared to those with a normal outcome (25.4 compared with $16.8, P<.01)$.

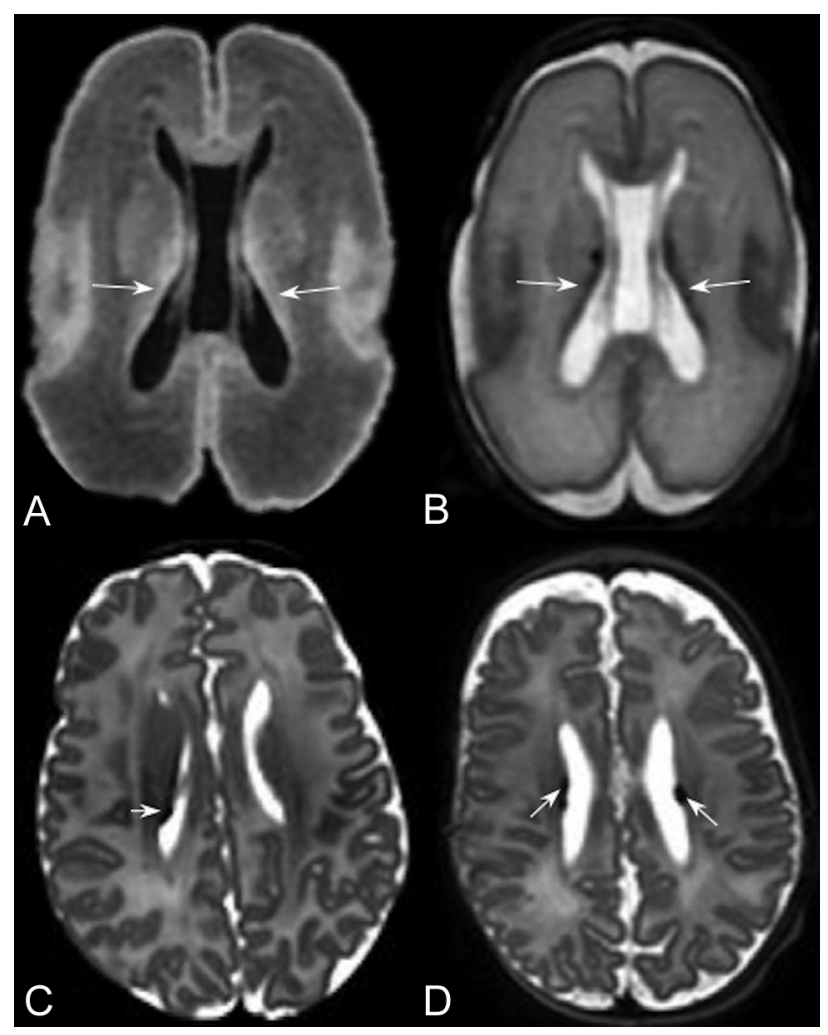

FIG 4. Germinal matrix. T1- $(A)$ and T2-weighted images $(B)$ of an infant born at $24+2$ weeks and imaged at $26+7$ weeks, demonstrating normal appearance of the germinal matrix for this gestation, here seen as a thin rim of low-T2/high-T7 signal in the subependymal region (arrows, $A$ and $B$ ). Germinal matrix hemorrhage is a complication of prematurity and is distinguished from normal matrix remnants by its shape and size. C, An infant born at $26+6$ weeks and imaged at $42+3$ weeks. $D$, An infant born at 28 weeks and imaged at $44+5$ weeks. The germinal matrix hemorrhage is recognized here as a thin, irregular focus of $\mathrm{T} 2$ low signal isolated to the subependyma, which may be unilateral (arrows, $C$ and $D$ ).

\section{Outcome and MR Imaging: GMH-IVH}

Fourteen percent of the cohort had GMH-IVH, 81\% of whom had concurrent lesions, $19 \%$ of which were major, and have been excluded from this analysis. Five percent of infants with GMHIVH had a GMFCS of $\geq 2$; none of these had abnormal myelin within the PLIC. Twenty-two percent had an abnormal motor score.

Infants with GMH-IVH and poorer outcomes were born earlier (gestational age, 27.5 versus 29.0 weeks, $P=.02$ ), with lower birth weight (1028 versus $1243 \mathrm{~g}, P=.04$ ) and higher IMD scores (22.2 versus $16.8, P=.05$ ) than those with normal outcomes.

\section{Outcome and MR Imaging: Hemorrhagic Parenchymal Infarction}

Three percent of infants had HPI, 47\% of whom had a GMFCS of $\geq 2$, and significantly more had abnormal motor and cognitive scores than those infants with no lesions $(P<.001)$. Thirty-three percent $(n=5)$ had normal development at 20 months, of whom $60 \%(n=3 / 5)$ had normal myelin within the PLIC.

\section{Outcome and MR Imaging: PVL}

Three percent of our cohort had PVL, all of whom had abnormal motor outcomes and a GMFCS of $\geq 2$. Ninety-two percent ( $n=$ 12/13) had accompanying asymmetry or abnormal signal within the PLIC. The presence of PVL was highly specific for abnormal motor and cognitive outcomes (Table).

\section{Outcome and MR Imaging: Punctate White Matter Lesions}

Punctate white matter lesions represented the most common lesion type in our cohort of preterm infants (41\%); $65 \%$ had additional lesions. After exclusion of those cases with major lesions, $63 \%$ of infants with PWML (any number) were developing normally at 20 months. Three percent of infants with $<10$ PWML and $6 \%$ of those with $>10$ PWML had a GMFCS of $\geq 2$. Infants with PWML from this cohort have been comprehensibly reported by Tusor et al, ${ }^{6}$ who, similarly, found a positive correlation increase in the risk of poor motor outcome; $>20$ PWML were $57 \%$ sensitive and $94 \%$ specific for a GMFCS of $\geq 2$.

Sensitivity, specificity, positive predictive value, and negative predictive value of lesions in isolation ${ }^{\text {a }}$ for BSID-III outcomes

\begin{tabular}{|c|c|c|c|c|c|c|c|c|c|c|c|c|}
\hline \multirow[b]{2}{*}{ Lesion $^{\mathrm{b}}$} & \multicolumn{4}{|c|}{$\begin{array}{c}\text { Abnormal Motor } \\
\text { Outcome }\end{array}$} & \multicolumn{4}{|c|}{$\begin{array}{c}\text { Abnormal Cognitive } \\
\text { Outcome }\end{array}$} & \multicolumn{4}{|c|}{$\begin{array}{c}\text { Abnormal Language } \\
\text { Outcome }\end{array}$} \\
\hline & SENS & SPEC & PPV & $\overline{\mathrm{NPV}}$ & SENS & SPEC & PPV & $\overline{\text { NPV }}$ & SENS & SPEC & PPV & $\overline{N P V}$ \\
\hline Any lesion present $(n=363)$ & 90 & 26 & 17 & 94 & 82 & 26 & 25 & 83 & 78 & 25 & 37 & 66 \\
\hline GMH-IVH $(n=54)$ & 63 & 72 & 22 & 94 & 41 & 70 & 24 & 83 & 37 & 71 & 43 & 66 \\
\hline Cerebellar hemorrhage $<5 \mathrm{~mm}(n=112)$ & 67 & 52 & 13 & 94 & 56 & 52 & 21 & 83 & 50 & 51 & 35 & 66 \\
\hline Cerebellar hemorrhage $>5 \mathrm{~mm}(n=7)$ & - & - & - & - & 5 & 94 & 14 & 83 & 3 & 93 & 14 & 66 \\
\hline Subependymal cysts $(n=133)$ & 68 & 47 & 11 & 94 & 64 & 49 & 25 & 83 & 59 & 49 & 42 & 66 \\
\hline $\mathrm{HPI}(n=15)$ & 56 & 95 & 60 & 94 & 32 & 94 & 60 & 83 & 19 & 93 & 60 & 66 \\
\hline $\operatorname{PVL}(n=13)$ & 65 & 100 & 100 & 94 & 37 & 98 & 85 & 83 & 15 & 93 & 54 & 66 \\
\hline Minor unclassified $(n=13)$ & - & - & - & - & 14 & 91 & 23 & 83 & 13 & 92 & 46 & 66 \\
\hline Major unclassified $(n=8)$ & 36 & 96 & 50 & 94 & 17 & 96 & 50 & 83 & 9 & 95 & 50 & 66 \\
\hline Abnormal myelin ( $n=47)$ & 38 & 95 & 55 & 90 & 24 & 94 & 55 & 81 & 14 & 92 & 51 & 65 \\
\hline
\end{tabular}

Note:-SENS indicates sensitivity; SPEC, specificity; PPV, positive predictive value; NPV, negative predictive value; -, no cases.

a "Isolated" implies major lesions excluded; there may be concurrent minor lesions.

${ }^{\mathrm{b}}$ Statistics are calculated with respect to the group with no focal lesion. 


\section{Outcome and MR Imaging: Subependymal Cysts}

Thirty-three percent of our cohort had subependymal cysts, $21 \%$ of whom had evidence of previous hemorrhage at TEA, though our protocol did not include a blood-sensitive sequence.

Thirteen percent of infants with subependymal cysts had major lesions; $54 \%$ of infants with subependymal cysts, in the absence of a major concurrent lesion, had normal neurodevelopment. Five percent of these infants had a GMFCS of two, 50\% of whom had concurrent low-grade GMH-IVH. None had abnormal myelin.

Infants with isolated subependymal cysts and abnormal outcomes were more likely to be male (67\% versus $43 \%, P=.02$ ), have higher IMD scores (24.9 versus $19.9, P=.02)$. and were ventilated for longer (6.0 days versus 2.9 days, $P=.02$ ) than infants with normal outcomes.

\section{Outcome and MR Imaging: Cerebellar Hemorrhage}

Cerebellar hemorrhage was seen in 30\% of the infants; $77 \%$ had additional lesions. Three percent $(n=7)$ of infants had isolated large cerebellar hemorrhage, $86 \%(n=6 / 7)$ had normal neurodevelopment at 20 months, and one had abnormal cognitive and language scores.

Of the $22 \%$ ( $n=112$ ) of infants with small cerebellar hemorrhage, none had a GMFCS of $\geq 2$ and $61 \%$ had normal neurodevelopment at 20 months. Infants with small cerebellar hemorrhages and an abnormal outcome were more likely to have higher IMD scores $(22.3$ versus $15.8, P<.01)$ and were imaged at a later PMA (43.5 versus 42.5 weeks, $P<.01)$ than those with normal outcomes.

\section{Outcome and MR Imaging: Unclassified Lesions}

Six percent $(n=30)$ of infants had unclassified lesions (On-line Table 3); $93 \%$ had a concomitant lesion, $53 \%$ of which were major. Thirteen infants with nonmajor unclassified lesions attended for assessment, of whom 54\% had normal neurodevelopment at 20 months. One infant had a GMFCS of 2, but none had an abnormal BSID-III motor score.

\section{Corpus Callosal Thinning and Ventriculomegaly}

Twenty-one percent $(n=106)$ of all participants had ventricular dilation, severe in $1 \%$. Eighty-four percent $(n=424)$ had corpus callosal thinning, classified as global thinning in $21 \%$. When we examined the infants with no focal lesions, only $17 \%$ had neither corpus callosal thinning nor ventriculomegaly; however, neither were independently associated with an adverse outcome in this subgroup (On-line Table 4).

\section{Neuropsychiatric Outcomes}

Eighty-seven percent $(n=445)$ of infants had parentally completed M-CHAT questionnaires, of whom 31\% were deemed high risk. Ninety-two percent $(n=12 / 13)$ of infants with PVL and $57 \%(n=8 / 14)$ with HPI had a high-risk M-CHAT score. The infants with high-risk M-CHAT scores were significantly more likely to have abnormal BSID-III cognitive (44\% versus $15 \%, P<.001)$ and motor (38\% versus $5 \%, P<.001)$ scores than those with a low-risk M-CHAT score.

\section{DISCUSSION}

We present $3 \mathrm{~T}$ MR brain imaging findings in a large, prospectively recruited, unselected cohort of preterm infants, born in 14 centers before 33 weeks' gestation and imaged in a single center, with $93 \%$ of infants returning for neurodevelopmental assessment at 20 months. Clear descriptions of the spectrum of lesions encountered alongside simple descriptive statistics will aid in clinical radiological interpretation for individual infants, without the use of scoring systems.

Acquired lesions are common in preterm born infants routinely imaged at TEA and were detected in $76 \%$ of infants in our cohort. We demonstrated that 3T MR imaging of the brain at TEA with no focal lesion has a sensitivity of $45 \%$ (95\% CI, 40\%-50\%) and a specificity of $61 \%(95 \% \mathrm{CI}, 51 \%-71 \%)$ for normal neurodevelopmental outcome (across all BSID-III domains) at 20 months and a sensitivity of $17 \%(95 \%$ CI, $14 \%-22 \%)$ and specificity of $94 \%$ (95\% CI, 88\%-97\%) for a normal motor outcome when used routinely in an unselected preterm population. None of the infants without focal lesions had a GMFCS of $\geq 2$. In contrast, motor impairment was common in infants with major lesions.

Only $3 \%$ of our cohort had PVL, and a further $3 \%$ had HPI, which is consistent with recent studies. ${ }^{7,8}$ All infants with PVL had abnormal motor outcomes. Outcomes in HPI were more variable, a finding consistent with other studies and meta-analyses. ${ }^{16}$ Abnormal myelination within the PLIC has been associated with the development of cerebral palsy in infants with HPI and termborn infants with hypoxic-ischemic encephalopathy. ${ }^{17,18}$ De Vries et $\mathrm{al}^{17}$ found HPI associated with abnormal signal in the PLIC to have a $100 \%$ positive predictive value for later hemiplegia, whereas we found abnormal myelination, in the context of HPI, to have a $60 \%$ positive predictive value for a gross motor impairment, but an $80 \%$ negative predictive value. We did not, however, include a neurological examination and may, therefore, have missed an asymmetry in tone with no functional deficit at the assessment at 20 months.

PWML were the most common lesion and have previously been extensively studied in this cohort by Tusor et al, ${ }^{6}$ who found a positive correlation increase in the risk of poor motor outcomes at 20 months with increments in the number of PWML seen on 3T MR imaging at TEA. More than 20 PWML were found to be $57 \%$ sensitive and $94 \%$ specific for a GMFCS of $\geq 2$, with a positive predictive value of 0.27 and negative predictive value of $0.98 .^{6}$

Opinion is divided on the effects of cerebellar hemorrhage on neurodevelopment, with heterogeneity of definitions and cohorts adding to the confusion. Few infants in our cohort had major cerebellar hemorrhages compared with other studies; ${ }^{19,20}$ however, $27 \%$ had minor hemorrhages and none had a GMFCS of $\geq 2$. Kidokoro et $\mathrm{al}^{19}$ used both $1.5 \mathrm{~T}$ and $3 \mathrm{~T}$ scanners and found similarly high rates of cerebellar hemorrhage in the cohort scanned at $3 \mathrm{~T}$ ( $23 \%$ versus $5 \%$ ).

We found no independent association between qualitative assessment of ventricular dilation and corpus callosal thinning and 20-month outcomes in the absence of a focal lesion. While another study found no association with quantitative corpus callosal volume and 2-year outcomes, ${ }^{21}$ others have found associations. ${ }^{22}$ Ventriculomegaly diagnosed by cranial sonography has 
been extensively studied, often in the context of intraventricular hemorrhage. More recently, some studies have explored a link between isolated ventriculomegaly in preterm infants and poor neurodevelopmental outcomes; ${ }^{23}$ however, MR imaging data are lacking. Our data suggest that qualitatively assessed corpus callosal thinning and ventricular dilation, in the absence of other acquired lesions, could be considered part of the preterm phenotype.

Seven infants in this study scored a GMFCS of 2 in the absence of a major lesion. While we were not able to exclude other potential causes of motor impairment in this study, other studies report similar small subgroups with abnormal outcomes but no imaging correlate. ${ }^{7,19}$ Three of these infants had isolated GMH$\mathrm{IVH}$, and while this only represents $5 \%$ of all GMH-IVH, there is growing evidence to suggest that these injuries are not benign. ${ }^{24}$ Postmortem human and animal studies examining the effect of germinal matrix hemorrhage have shown that the presence of blood in the germinal matrix suppresses germinal cell line proliferation; ${ }^{25}$ this finding is further supported by a reduction in cortical volume on MR imaging in preterm infants with uncomplicated GMH-IVH, ${ }^{26}$ which may explain, in part, the occasional poorer outcomes in this population.

For this study, we accept the limitation of a relatively young follow-up age and that the GMFCS score of 1 did not discriminate between normal development and mild impairment, and a score of 2 is not necessarily discriminatory for cerebral palsy. Both GMFCS and BSID-III will likely miss milder tone abnormalities, which may evolve to cause significant motor impairment; and it is not inconceivable that a child with hemiplegia could have scores within the normal range. A subgroup of infants had abnormal BSID-III motor scores in the absence of a major lesion; however, some studies have advised that a threshold of 85 may overestimate motor impairment. ${ }^{27}$ We accept that there may be alternative causes for any motor impairment or more subtle changes for which advanced MR imaging techniques may offer more insight. Extended follow-up, now in progress, will be of interest in these cases.

Thirty-one percent of our cohort had an abnormal MCHAT score; however, a significant proportion had concomitant abnormal motor and cognitive BSID-III scores. These findings are comparable with those of Moore et al, ${ }^{28}$ who highlighted the difficulties in using M-CHAT in preterm infants who are at high risk of motor and cognitive impairment. It is, therefore, likely that some of our results reflect cognitive and other dysfunctions rather than a specific diagnosis of autism spectrum disorder.

We also found that an increasing IMD score, a measure of adverse socioeconomic status, was associated with poorer neurodevelopmental outcomes; this finding supports evidence linking socioeconomic status and maternal education to early neurodevelopment and, specifically, cognition: ${ }^{29,30}$ this is potentially encouraging as a modifiable parameter and is corroborated by studies offering social interventions to children from lower income families. $^{31}$

\section{CONCLUSIONS}

Acquired brain lesions are common in preterm neonates routinely undergoing 3T MR imaging at term-equivalent age. Major lesions (ie, HPI and PVL) confer a poor neurodevelopmental prognosis; conversely, infants without acquired lesions all had normal gross motor function (GMFCS of $<2$ ) at 20 months.

\section{ACKNOWLEDGMENTS}

We are very grateful to the families, clinicians, and investigators who made the ePrime study possible.

Disclosures: Sophie Arulkumaran—RELATED: Grant: Medical Research Council, Guys and St. Thomas' Charity, National Institute of Health Research Program Grant, Comments: Medical Research Council, code MR/K006355/1, Guys and St Thomas' Charity (code STR1110011), National Institute for Health Research Program Grant (code RP-PG-0707-10154).* Shona Falconer-RELATED: Grant: European Research Council, Comments: Synergy FP7 (Developing Human Connectome Project).* Joseph V. Hajnal-RELATED: Grant: European Research Council, Comments: funding to acquire data.* Serena J. Counsell-RELATED: Grant: National Institute for Health Research, Comments: National Institute for Health Research Program Grants for Applied Research Program (RP-PG-070710154)*; UNRELATED: Employment: Professor at King's College London. Mary A. Rutherford-RELATED: Grant: National Institute of Health Research, Comments: E-Prime study funded by the National Institute for Health Research. Anthony D. Edwards_RELATED: Grant: National Institute for Health Research, Comments: National Institute for Health Research Applied Program Grant funded the data acquisition. * *Money paid to the institution.

\section{REFERENCES}

1. Beck S, Wojdyla D, Say L, et al. The worldwide incidence of preterm birth: a systematic review of maternal mortality and morbidity. Bull World Health Organ 2010;88:31-38 CrossRef Medline

2. Costeloe KL, Hennessy EM, Haider S, et al. Short term outcomes after extreme preterm birth in England: comparison of two birth cohorts in 1995 and 2006 (the EPICure studies). BMJ 2012;345: e7976 CrossRef Medline

3. Deng W, Pleasure J, Pleasure D. Progress in periventricular leukomalacia. Arch Neurol 2008;65:1291-95 CrossRef Medline

4. Edwards AD, Redshaw ME, Kennea N, et al; ePrime Investigators. Effect of MRI on preterm infants and their families: a randomised trial with nested diagnostic and economic evaluation. Arch Dis Child Fetal Neonatal Ed 2018;103:F15-21 CrossRef Medline

5. Counsell SJ, Edwards AD, Chew AT, et al. Specific relations between neurodevelopmental abilities and white matter microstructure in children born preterm. Brain 2008;131:3201-08 CrossRef Medline

6. Tusor N, Benders MJ, Counsell SJ, et al. Punctate white matter lesions associated with altered brain development and adverse motor outcome in preterm infants. Sci Rep 2017;7:13250 CrossRef Medline

7. Hintz SR, Barnes PD, Bulas D, et al; SUPPORT Study Group of the Eunice Kennedy Shriver National Institute of Child Health and Human Development Neonatal Research Network. Neuroimaging and neurodevelopmental outcome in extremely preterm infants. Pediatrics 2015;135:e32-42 CrossRef Medline

8. Woodward LJ, Anderson PJ, Austin NC, et al. Neonatal MRI to predict neurodevelopmental outcomes in preterm infants. $N$ Engl J Med 2006;355:685-94 CrossRef Medline

9. Tam EW, Rosenbluth G, Rogers EE, et al. Cerebellar hemorrhage on magnetic resonance imaging in preterm newborns associated with abnormal neurologic outcome. J Pediatr 2011;158:245-50 CrossRef Medline

10. Rutherford MA, ed. MRI of the Neonatal Brain. 4th ed. Saunders; 2002

11. Srinivasan L, Dutta R, Counsell SJ, et al. Quantification of deep gray matter in preterm infants at term-equivalent age using manual volumetry of 3-Tesla magnetic resonance images. Pediatrics 2007; 119:759-65 CrossRef Medline

12. Cooper S, Bar-Yosef O, Berkenstadt M, et al. Prenatal evaluation, imaging features, and neurodevelopmental outcome of prenatally diagnosed periventricular pseudocysts. AJNR Am J Neuroradiol 2016;37:2382-88 CrossRef Medline 
13. Bayley N. Bayley Scales of Infant and Toddler Development Screening Test. 3rd ed. Psychological Corporation; 2006

14. Robins DL, Fein D, Barton ML, et al. The Modified Checklist for Autism in Toddlers: an initial study investigating the early detection of autism and pervasive developmental disorders. J Autism Dev Disord 2001;31:131-44 CrossRef Medline

15. Johnson S, Moore T, Marlow N. Using the Bayley-III to assess neurodevelopmental delay: which cut-off should be used? Pediatr Res 2014;75:670-74 CrossRef Medline

16. Nongena P, Ederies A, Azzopardi DV, et al. Confidence in the prediction of neurodevelopmental outcome by cranial ultrasound and MRI in preterm infants. Arch Dis Child Fetal Neonatal Ed 2010;95: F388-90 CrossRef Medline

17. De Vries LS, Groenendaal F, van Haastert IC, et al. Asymmetrical myelination of the posterior limb of the internal capsule in infants with periventricular haemorrhagic infarction: an early predictor of hemiplegia. Neuropediatrics 1999;30:314-19 CrossRef Medline

18. Rutherford MA, Pennock JM, Counsell SJ, et al. Abnormal MRI signal in the internal capsule predicts poor neurodevelopmental outcome in infants with hypoxic-ischaemic encephalopathy. Pediatrics 1998;102:323-28 CrossRef Medline

19. Kidokoro H, Anderson PJ, Doyle LW, et al. Brain injury and altered brain growth in preterm infants: predictors and prognosis. Pediatrics 2014;134:e444-53 CrossRef Medline

20. Hintz SR, Vohr BR, Bann CM, et al; SUPPORT study group of the Eunice Kennedy Shriver National Institute of Child Health and Human Development Neonatal Research Network. Preterm neuroimaging and school-age cognitive outcomes. Pediatrics 2018;142: e20174058 CrossRef Medline

21. Thompson DK, Inder TE, Faggian N, et al. Corpus callosum alterations in very preterm infants: perinatal correlates and 2-year neurodevelopmental outcomes. Neuroimage 2012;59:3571-81 CrossRef Medline
22. Rademaker KJ, Lam J, Van Haastert IC, et al. Larger corpus callosum size with better motor performance in prematurely born children. Semin Perinatol 2004;28:279-87 CrossRef Medline

23. Pappas A, Adams-Chapman I, Shankaran S, et al; Eunice Kennedy Shriver National Institute of Child Health and Human Development Neonatal Research Network. Neurodevelopmental and behavioral outcomes in extremely premature neonates with ventriculomegaly in the absence of periventricular-intraventricular hemorrhage. JAMA Pediatr 2018;172:32-42 CrossRef Medline

24. Patra K, Wilson-Costello D, Taylor HG, et al. Grades I-II intraventricular hemorrhage in extremely low birth weight infants: effects on neurodevelopment. J Pediatr 2006;149:169-73 CrossRef Medline

25. Del Bigio MR. Cell proliferation in human ganglionic eminence and suppression after prematurity-associated haemorrhage. Brain 2011;134:1344-61 CrossRef Medline

26. Vasileiadis GT, Gelman N, Han VKM, et al. Uncomplicated intraventricular hemorrhage is followed by reduced cortical volume at near-term age. Pediatrics 2004;114:e367-72 CrossRef Medline

27. Duncan AF, Bann C, Boatman C, et al. Do currently recommended Bayley-III cutoffs overestimate motor impairment in infants born $<27$ weeks gestation? J Perinatol 2015;35:516-21 CrossRef Medline

28. Moore T, Hennessy EM, Myles J, et al. Neurological and developmental outcome in extremely preterm children born in England in 1995 and 2006: the EPICure studies. BMJ 2012;345:e7961 CrossRef Medline

29. Hackman DA, Farah MJ. Socioeconomic status and the developing brain. Trends Cogn Sci 2009;13:65-73 CrossRef Medline

30. Benavente-Fernández I, Siddiqi A, Miller SP. Socioeconomic status and brain injury in children born preterm: modifying neurodevelopmental outcome. Pediatr Res 2020;87:391-98 CrossRef Medline

31. Olds DL, Holmberg JR, Donelan-McCall N, et al. Effects of home visits by paraprofessionals and by nurses on children: follow-up of a randomized trial at ages 6 and 9 years. JAMA Pediatr 2014;168:114 CrossRef Medline 